\title{
Publisher Correction: Genetic analysis of over 1 million people identifies 535 new loci associated with blood pressure traits
}

Evangelos Evangelou (D, Helen R. Warren, David Mosen-Ansorena, Borbala Mifsud, Raha Pazoki, He Gao, Georgios Ntritsos, Niki Dimou, Claudia P. Cabrera, Ibrahim Karaman (10), Fu Liang Ng, Marina Evangelou, Katarzyna Witkowska, Evan Tzanis, Jacklyn N. Hellwege $\mathbb{D}^{0}$, Ayush Giri D, Digna R. Velez Edwards, Yan V. Sun, Kelly Cho, J. Michael Gaziano, Peter W. F. Wilson,

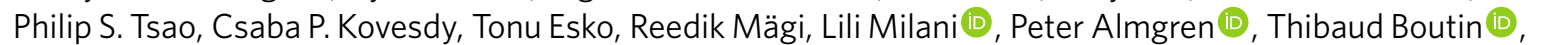
Stéphanie Debette, Jun Ding, Franco Giulianini, Elizabeth G. Holliday, Anne U. Jackson (1D, Ruifang Li-Gao, Wei-Yu Lin (1), Jian'an Luan, Massimo Mangino (1D), Christopher Oldmeadow, Bram Peter Prins, Yong Qian, Muralidharan Sargurupremraj, Nabi Shah (D), Praveen Surendran, Sébastien Thériault, Niek Verweij, Sara M. Willems, Jing-Hua Zhao, Philippe Amouyel (1), John Connell, Renée de Mutsert, Alex S. F. Doney, Martin Farrall, Cristina Menni®, Andrew D. Morris, Raymond Noordam, Guillaume Paré, Neil R. Poulter, Denis C. Shields, Alice Stanton (1D, Simon Thom Đ), Gonçalo Abecasis, Najaf Amin, Dan E. Arking, Kristin L. Ayers, Caterina M. Barbieri, Chiara Batini, Joshua C. Bis, Tineka Blake, Murielle Bochud, Michael Boehnke (D), Eric Boerwinkle, Dorret I. Boomsma, Erwin P. Bottinger, Peter S. Braund, Marco Brumat (D), Archie Campbell(1), Harry Campbell, Aravinda Chakravarti, John C. Chambers, Ganesh Chauhan, Marina Ciullo (D), Massimiliano Cocca (1), Francis Collins (1D), Heather J. Cordell, Gail Davies, Martin H. de Borst (D), Eco J. de Geus (D), Ian J. Deary, Joris Deelen (D), Fabiola Del Greco M., Cumhur Yusuf Demirkale, Marcus Dörr, Georg B. Ehret (1), Roberto Elosua, Stefan Enroth (1), A. Mesut Erzurumluoglu (1), Teresa Ferreira, Mattias Frånberg, Oscar H. Franco, Ilaria Gandin, Paolo Gasparini, Vilmantas Giedraitis (D), Christian Gieger, Giorgia Girotto $\mathbb{D}$, Anuj Goel (D), Alan J. Gow (D), Vilmundur Gudnason, Xiuqing Guo, Ulf Gyllensten, Anders Hamsten, Tamara B. Harris, Sarah E. Harris, Catharina A. Hartman, Aki S. Havulinna (D), Andrew A. Hicks (1D, Edith Hofer, Albert Hofman, Jouke-Jan Hottenga,

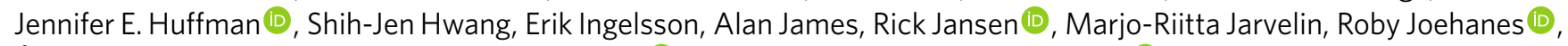
Åsa Johansson, Andrew D. Johnson, Peter K. Joshi@ , Pekka Jousilahti, J. Wouter Jukema®D, Antti Jula, Mika Kähönen, Sekar Kathiresan, Bernard D. Keavney D , Kay-Tee Khaw, Paul Knekt, Joanne Knight $\mathbb{D}$, Ivana Kolcic, Jaspal S. Kooner, Seppo Koskinen, Kati Kristiansson (1), Zoltan Kutalik (1), Maris Laan, Marty Larson, Lenore J. Launer, Benjamin Lehne, Terho Lehtimäki, David C. M. Liewald (1), Li Lin, Lars Lind, Cecilia M. Lindgren, YongMei Liu, Ruth J. F. Loos (i),

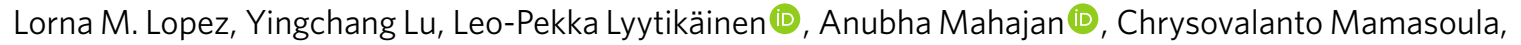
Jaume Marrugat, Jonathan Marten, Yuri Milaneschi, Anna Morgan (D), Andrew P. Morris, Alanna C. Morrison, Peter J. Munson, Mike A. Nalls, Priyanka Nandakumar, Christopher P. Nelson, Teemu Niiranen, Ilja M. Nolte (D, Teresa Nutile (1), Albertine J. Oldehinkel (D), Ben A. Oostra, Paul F. O'Reilly @, Elin Org, Sandosh Padmanabhan, Walter Palmas, Aarno Palotie, Alison Pattie, Brenda W. J. H. Penninx, Markus Perola, Annette Peters, Ozren Polasek, Peter P. Pramstaller, Quang Tri Nguyen, Olli T. Raitakari, Meixia Ren, Rainer Rettig, Kenneth Rice, Paul M. Ridker, Janina S. Ried, Harriëtte Riese, Samuli Ripatti, Antonietta Robino, Lynda M. Rose, Jerome I. Rotter, Igor Rudan (D, Daniela Ruggiero, Yasaman Saba, Cinzia F. Sala, Veikko Salomaa (D), Nilesh J. Samani, Antti-Pekka Sarin, Reinhold Schmidt, Helena Schmidt, Nick Shrine (D), David Siscovick, Albert V. Smith —D, Harold Snieder, Siim Sõber (D), Rossella Sorice, John M. Starr, David J. Stott, David P. Strachan (1), Rona J. Strawbridge, Johan Sundström (D, Morris A. Swertz (D, Kent D. Taylor, Alexander Teumer (D), Martin D. Tobin D, Maciej Tomaszewski, Daniela Toniolo, Michela Traglia, Stella Trompet, Jaakko Tuomilehto, Christophe Tzourio, André G. Uitterlinden, Ahmad Vaez (1), Peter J. van der Most (1D, Cornelia M. van Duijn, Anne-Claire Vergnaud, Germaine C. Verwoert, Veronique Vitart, Uwe Völker, Peter Vollenweider, Dragana Vuckovic, Hugh Watkins, Sarah H. Wild, Gonneke Willemsen, James F. Wilson (1), Alan F. Wright, Jie Yao, Tatijana Zemunik (1), Weihua Zhang, John R. Attia (1), Adam S. Butterworth (D), Daniel I. Chasman, David Conen, Francesco Cucca, John Danesh, Caroline Hayward D, Joanna M. M. Howson (1D, Markku Laakso, Edward G. Lakatta, Claudia Langenberg, Olle Melander, Dennis O. Mook-Kanamori, Colin N. A. Palmer (D, Lorenz Risch, Robert A. Scott, Rodney J. Scott, Peter Sever, Tim D. Spector, Pim van der Harst (1D, Nicholas J. Wareham, Eleftheria Zeggini (i), Daniel Levy, Patricia B. Munroe (D), Christopher Newton-Cheh, Morris J. Brown, Andres Metspalu, Adriana M. Hung, Christopher J. O'Donnell, Todd L. Edwards (D), the Million Veteran Program, Bruce M. Psaty, loanna Tzoulaki (D, Michael R. Barnes (1), Louise V. Wain (1), Paul Elliott 1 and Mark J. Caulfield (1D)

Correction to: Nature Genetics https://doi.org/10.1038/s41588-018-0205-x, published online 17 September 2018.

In the version of this article originally published, the name of author Martin H. de Borst was coded incorrectly in the XML. The error has now been corrected in the HTML version of the paper.

Published online: 14 November 2018

https://doi.org/10.1038/s41588-018-0297-3 